\title{
Quantitative Trait Loci from Two Genotypes of Oat (Avena sativa) Conditioning Resistance to Puccinia coronata
}

\author{
Ebrahiem M. Babiker, Tyler C. Gordon, Eric W. Jackson, Shiaoman Chao, \\ Stephen A. Harrison, Martin L. Carson, Don E. Obert, and J. Michael Bonman
}

First, second, and eighth authors: USDA-ARS, Small Grains and Potato Germplasm Research Unit, Aberdeen, ID 83210; third author: General Mills Inc., 150 Research Drive, Kannapolis, NC 28081; fourth author: USDA-ARS, Cereal Crops Research, 1605 Albrecht Blvd., Fargo, ND 58102; fifth author: Louisiana State University Agricultural Center, 104 M.B. Sturgis Hall, Baton Rouge, LA 70803; sixth author: USDA-ARS, Cereal Disease Laboratory, St. Paul 55108; and seventh author: Limagrain Cereal Seeds, 9020 Grant Road, Battle Ground, IN 47920.

Accepted for publication 6 August 2014.

\begin{abstract}
Babiker, E. M., Gordon, T. C., Jackson, E. W., Chao, S., Harrison, S. A., Carson, M. L., Obert, D. E., and Bonman, J. M. 2015. Quantitative trait loci from two genotypes of oat (Avena sativa) conditioning resistance to Puccinia coronata. Phytopathology 105:239-245.

Developing oat cultivars with partial resistance to crown rust would be beneficial and cost-effective for disease management. Two recombinant inbred-line populations were generated by crossing the susceptible cultivar Provena with two partially resistant sources, CDC Boyer and breeding line 94197A1-9-2-2-2-5. A third mapping population was generated by crossing the partially resistant sources to validate the quantitative trait locus (QTL) results. The three populations were evaluated for crown rust

the 2009 CDL test, linkage analyses revealed two QTLs for partial resistance in the Provena/CDC Boyer population on chromosome 19A. One of the 19A QTLs was also detected in the 2009 LSU test. Another QTL was detected on chromosome 12D in the CDL 2009 test. In the Provena/ 94197A1-9-2-2-2-5 population, only one QTL was detected, on chromosome 13A, in the CDL 2011 test. The 13A QTL from the Provena/ 94197A1-9-2-2-2-5 population was validated in the CDC Boyer/94197A19-2-2-2-5 population in the CDL 2010 and 2011 tests. Comparative analysis of the significant marker sequences with the rice genome database revealed 15 candidate genes for disease resistance on chromosomes 4 and 6 of rice. These genes could be potential targets for cloning from the two resistant parents.
\end{abstract} severity in the field at Louisiana State University (LSU) in 2009 and 2010 and at the Cereal Disease Laboratory (CDL) in St. Paul, MN, in 2009, 2010, and 2011. An iSelect platform assay containing 5,744 oat single nucleotide polymorphisms was used to genotype the populations. From
Additional keywords: oat resistance, Puccinia coronata, single nucleotide polymorphism.
Crown rust, caused by Puccinia coronata f. sp. avenae Eriks., is the most destructive disease of oat worldwide (18). Crown rust management has relied primarily on the use of race-specific crown rust resistance $(P c)$ genes derived from cultivated oat Avena sativa, wild oats $A$. sterilis and $A$. strigosa, and tetraploid Avena spp. $(6,17)$. More than $96 P c$ genes have been identified and many have been deployed, but resistance conferred by single $P c$ genes is short-lived $(6,17)$.

The use of partial resistance (PR) to crown rust, where a compatible host-pathogen interaction exists but disease is reduced compared to a fully susceptible host, has been proposed as a means to improve the durability of crown rust resistance $(5,12$, 17). Several studies have shown that $P R$ to oat crown rust can be detected in adult plants and is generally conditioned by several quantitative trait loci (QTLs), but these QTLs could not be assigned to specific chromosomes because the chromosome or physical map location of the molecular markers used in the studies was unknown $(3,9,16)$. In addition, few polymerase chain reaction-based markers were available for use in the previous mapping work, which slowed progress toward marker-assisted breeding for crown rust resistance. Recently, these drawbacks have been largely overcome with the development of a single

Corresponding author: J. M. Bonman; E-mail address: Mike.Bonman@ars.usda.gov

http://dx.doi.org/10.1094/PHYTO-04-14-0114-R

This article is in the public domain and not copyrightable. It may be freely reprinted with customary crediting of the source. The American Phytopathological Society, 2015 nucleotide polymorphism (SNP) marker platform for oat and a physically anchored hexaploid oat map with 21 chromosomes (13).

In a previous effort to identify PR in improved oat genotypes, 86 cultivars and breeding lines were evaluated in multiple field locations (United States and Canada) over 2 years (4). Several materials, including the cultivar CDC Boyer and the breeding line 94197A1-9-2-2-2-5, consistently displayed PR at the adult stage. CDC Boyer was developed by the University of Saskatchewan Crop Development Center and grown in Canada. Although the cultivar was infected by crown rust under Canadian production condition, it never showed economically damaging levels of disease (B. Rossnagel, personal communication). The breeding line 94197A1-9-2-2-2-5 was developed by Greg Shaner at Purdue University from a cross with A. sterilis. Because the PR of CDC Boyer was useful in practical agriculture and that in 94197A1-92-2-2-5 could be novel, these materials were chosen to develop PR mapping populations.

The quantitative nature of PR to crown rust and the fact that it is often evident only in adult plants makes phenotypic selection more complicated and time-consuming compared to selection for the complete resistance provided by $P c$ genes, which is easily identified at the seedling stage. To improve the efficiency of oat breeding for PR to crown rust, it is important to identify QTLs, determine their chromosomal locations, and develop tightly linked molecular markers to aid marker-assisted selection (MAS). SNP markers are now the markers of choice in oat genetic analysis (14). Compared to other molecular markers, SNP markers provide better genome coverage and are amenable for genotyping 
using high-throughput automated scoring platforms (14). The objectives of this study were to characterize crown rust resistance in CDC Boyer and 94197A1-9-2-2-2-5 by identifying (i) QTLs for PR to crown rust in adult plants based on five replicated field trials conducted under highly disease-conducive conditions and (ii) candidate resistance genes by using the oat cDNA sequence data from which the SNP markers were developed.

\section{MATERIALS AND METHODS}

Plant materials. Three oat mapping populations were used in this study. Two populations were generated by crossing the resistant sources, CDC Boyer and 94197A1-9-2-2-2-5, to the susceptible oat cultivar Provena (denoted as PB and P9 populations, respectively). The third population was developed by crossing 94197A1-9-2-2-2-5 to CDC Boyer (9B population). A total of $200 \mathrm{~F}_{6: 8}$ recombinant inbred lines (RILs) were derived through single-seed descent for each of the $\mathrm{PB}, \mathrm{P} 9$, and $9 \mathrm{~B}$ populations. CDC Boyer was released in 1994 by the Crop Development Centre, University of Saskatchewan. 94197A1-9-2-2-2-5 was developed at Purdue University (Pedigree: PI 539877/3/Brawn// Clint/PI 412163 [A. sterilis]). Provena was released cooperatively by USDA-ARS and the Idaho Agricultural Experiment Station in 2000 (8). Except for a high level of susceptibility to P. coronata at both seedling and adult plant stages, Provena has been one of the best-performing hulless oat varieties in the western United States (8).

Adult plant field evaluation. Parents and 200 RILs from each population were evaluated under natural disease pressure at the Louisiana State University (LSU) Central Research Station in Baton Rouge, LA, in 2009 and 2010 and at the USDA-ARS Cereal Disease Laboratory (CDL), in the Matt Moore Buckthorn Nursery, in St Paul, MN, in 2009, 2010, and 2011 for a total of five environments. The LSU trials were planted in mid-November of each year. Plots were first sown in two $2.44-\mathrm{m}$ rows with $0.36-\mathrm{m}$ spacing and were trimmed to approximately $1.2 \mathrm{~m}$ after emergence. Two rows of the crown-rust-susceptible cultivar Brooks were sown between each pair of test plots so that every test plot was adjacent to a two-row spreader plot. The CDL trials were planted in 1.2-m rows in April of each year, and buckthorn (Rhamnus cathartica), the alternative host of $P$. coronata in the United States, was maintained along the nursery perimeter to support the maximum sexual recombination of the fungus and to expose the nursery to high disease pressure from a highly diverse population of $P$. coronata. The experiments were arranged in a randomized complete block design with two replications and 10 plots of each parent randomly planted in each block. At the milk to early dough stage, the crown rust severity was evaluated visually on a wholeplot basis using the modified Cobb scale (15).

Molecular marker analysis. Out of the 200 RILs tested from each of the three populations, only the parents, 148 RILs from the PB population, 145 RILs from the P9 population, and 80 RILs from the 9B population were used for DNA extraction, following a protocol by Anderson et al. (2) with modification, including use of a bead grinder FastPrep homogenizer (MP Biomedical) for 5 min at 25 strokes per second. A newly developed iSelect SNP genotyping platform containing 5,744 oat SNP markers, based on an expansion of the work by Oliver et al. (13), was used to genotype parents and RILs from the three populations. Infinium genotyping assays developed by Illumina were performed as described by the manufacturer. Allele calling for each SNP was performed using Illumina's GenomeStudio software and was manually inspected for call accuracy.

Statistical analysis. Analysis of variance was carried out using the General Linear Model Procedure in SAS (SAS Institute, Cary, NC). The significance of genotype, location, and year was examined. The mean disease severity value for each genotyped RIL across replication within each experiment was calculated in SAS and used in QTL analysis.

Map construction and QTL analysis. Polymorphic markers with less than $9 \%$ missing data and that had previously been assigned to chromosomes were used in the analysis. A linkage map was constructed using JMP Genomics software Version 6 (SAS Institute, Cary, NC, 2012). The recombination and linkage groups and the linkage map order functions were used to determine the initial number of linkage groups and the most likely marker order. The interval mapping and composite interval mapping (IM-CIM) analysis function was used to generate a composite interval map of QTLs with a minimum logarithm of odds (LOD) threshold of 3.0. Genetic distances between markers were calculated in centiMorgans $(\mathrm{cM})$ using the Kosambi map function, and linkage groups were assigned to chromosomes based on the published oat consensus map (13).

Sequence homology. To identify candidate genes within the QTL regions, the sequences of the significant markers were compared for their homology with the rice (Oryza sativa L.) genome (http://rice.plantbiology.msu.edu) using Blastn and Blastx.

\section{RESULTS}

Phenotypic evaluation. Analysis of variance (ANOVA) indicated that the two resistant parents, CDC Boyer and 94197A1-

TABLE 1. Crown rust severity and mean square values from analysis of variance for three recombinant inbred line (RIL) populations of oat tested for crown rust resistance at the Cereal Disease Laboratory (CDL) in St. Paul, MN, and at Louisiana State University (LSU) in Baton Rouge, LA

\begin{tabular}{|c|c|c|c|c|c|c|c|c|}
\hline \multirow[b]{3}{*}{ Population } & \multirow[b]{3}{*}{ Environment } & \multicolumn{3}{|c|}{ Parent means } & \multirow{2}{*}{\multicolumn{2}{|c|}{ RILs }} & & \\
\hline & & \multirow[b]{2}{*}{ CDC-Boyer } & \multirow[b]{2}{*}{ Provena } & \multirow{2}{*}{$\begin{array}{l}94197 \mathrm{~A} 1- \\
9-2-2-2-5\end{array}$} & & & \multicolumn{2}{|c|}{ Mean square } \\
\hline & & & & & Mean & Range & Genotypes $^{\mathrm{a}}$ & Block $^{\mathrm{a}}$ \\
\hline \multirow{5}{*}{ Provena $\times$ CDC-Boyer } & CDL-09 & 40 & 89 & $\ldots$ & 77.3 & $32.5-90$ & $302.13^{* *}$ & $772.98 * *$ \\
\hline & CDL-10 & 76.6 & 90 & $\ldots$ & 86.7 & $40-95$ & $115.24 *$ & 210.21 \\
\hline & CDL-11 & 53.9 & 78.2 & $\ldots$ & 75.1 & $50.7-88$ & $130.11^{* *}$ & $159.1 *$ \\
\hline & LSU-09 & 30.7 & 79.6 & $\ldots$ & 48.5 & $5.1-97.5$ & $493.01 * *$ & $17,402.1 * *$ \\
\hline & LSU-10 & 47.6 & 92.4 & $\ldots$ & 60.6 & $2.5-97.5$ & $1,242.80 * *$ & $4748.56 * *$ \\
\hline \multirow{5}{*}{ Provena $\times 94197$ A1-9-2-2-2-5 } & CDL-09 & $\ldots$ & 89 & 37.5 & 68.9 & $32.5-90$ & $610.59 * *$ & $975.87 *$ \\
\hline & CDL-10 & $\ldots$ & 94 & 71.2 & 82.7 & $25-97$ & $148.39 * *$ & $711.26 * *$ \\
\hline & CDL-11 & $\ldots$ & 78.3 & 46.7 & 69.7 & $25-88$ & $594.36 * *$ & $337.89 *$ \\
\hline & LSU-09 & $\ldots$ & 65.6 & 13.4 & 32.8 & $1-90$ & $704.04 * *$ & $10,978.06 * *$ \\
\hline & LSU-10 & $\ldots$ & 87.2 & 49.2 & 71.1 & $2.5-97.5$ & $722.47 *$ & $61,851.6^{* *}$ \\
\hline \multirow{5}{*}{ CDC Boyer $\times$ 94197A1-9-2-2-2-5 } & CDL-09 & 40 & $\ldots$ & 37.5 & 50.7 & $20-90$ & $567.9 * *$ & $6,536.56 * *$ \\
\hline & CDL-10 & 76.6 & $\ldots$ & 62.5 & 72.7 & $25-5$ & $360.9 * *$ & $3,386.7 * *$ \\
\hline & CDL-11 & 53.7 & $\ldots$ & 46.7 & 58.2 & $22-88$ & $752.8 * *$ & $4,270.26^{* *}$ \\
\hline & LSU-09 & 24.9 & $\ldots$ & 11.4 & 18.6 & $1-80$ & $226.51 * *$ & $9,206.07 * *$ \\
\hline & LSU-10 & 48 & $\ldots$ & 42.2 & 42.2 & $1-90$ & 384.7 & $34,298.78 * *$ \\
\hline
\end{tabular}

a Asterisk (*) indicates significance at the 0.05 probability level, and ** indicates significance at the 0.001 probability level. 
9-2-2-2-5, were significantly different from the susceptible parent Provena $(P<0.05)$. Location, year, and genotype had significant effects on disease severity for the three populations $(P<0.05$; Table 1). In $\mathrm{PB}$ and $\mathrm{P} 9$ populations, transgressive segregation was observed, with several RILs exhibiting higher levels of disease severity in more than one trial compared to Provena (Figs. 1 and 2).

Mapping adult plant field resistance. Provena/CDC Boyer. Of 5,744 SNP markers, 899 polymorphic markers were used to construct a linkage map for the PB population. Of these 899 markers, 831 had previously been assigned to oat chromosomes and were used in the analysis. The 831 SNPs were assigned to 34 linkage groups representing 21 oat chromosomes with a total map length of $1,058.5 \mathrm{cM}$. QTL analyses were conducted for each of the five environments by using the average from the two replicates. Using the CIM function, three QTLs for PR to crown rust were detected in the PB population. Using data from the 2009 CDL test, two QTLs were detected on chromosome 19A and designated QCr.cdl9-19A and QCr.cdl9lsu9-19A, and one QTL designated QCr.cdl9-12D was detected on chromosome 12D. QCr.cdl9-19A was detected on chromosome 19A in a 10.6-cM

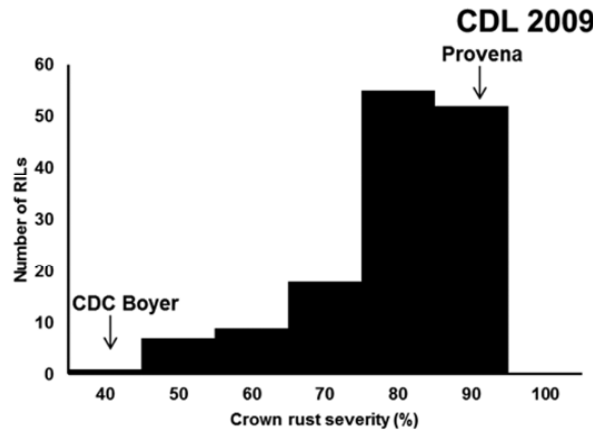

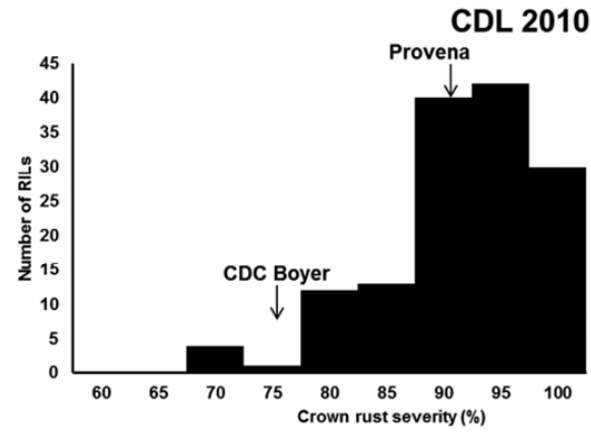

LSU 2009

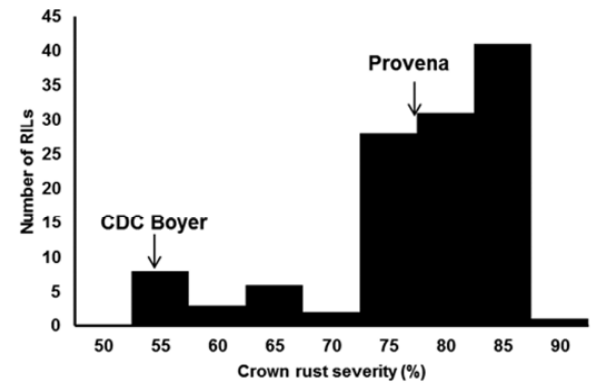

LSU 2010
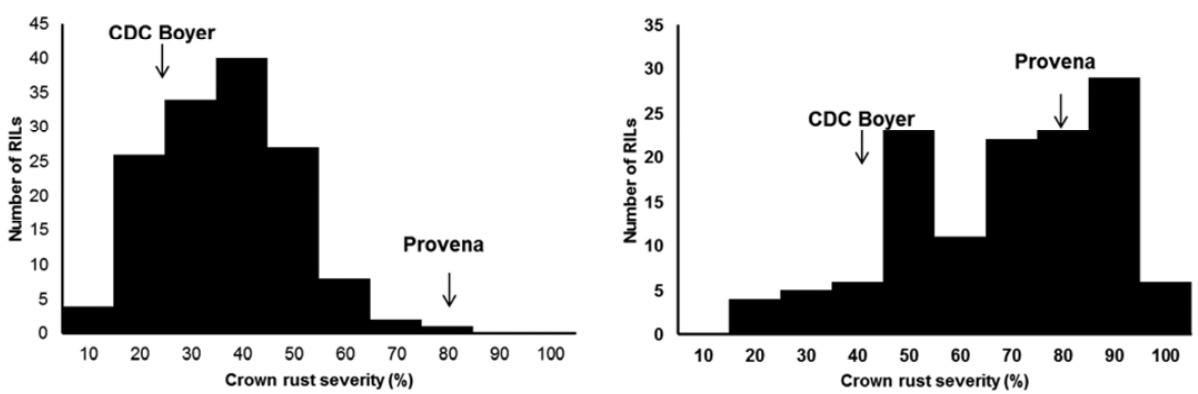

Fig. 1. Frequency distribution of oat crown rust severity in recombinant inbred lines (RILs) from a cross between Provena and CDC Boyer tested in five field trials conducted at the Cereal Disease Laboratory (CDL) in St. Paul, MN, and at Louisiana State University (LSU) in Baton Rouge, LA.

CDL 2009

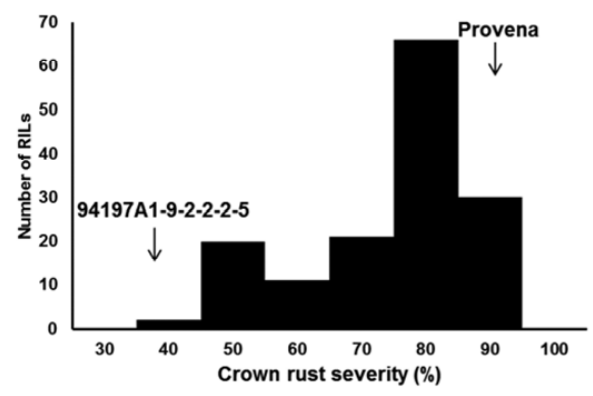

CDL 2010

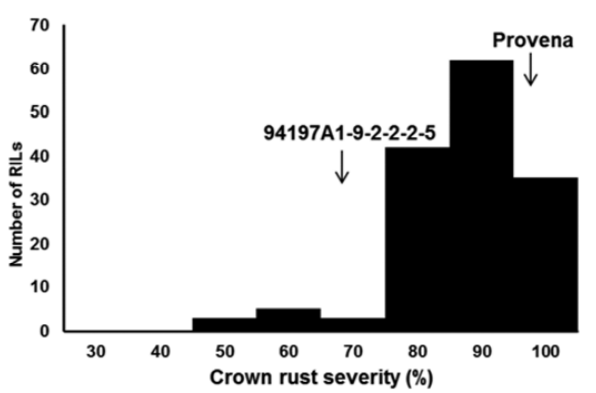

CDL 2011

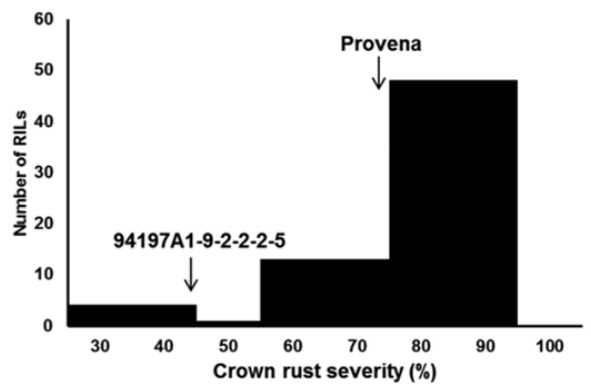

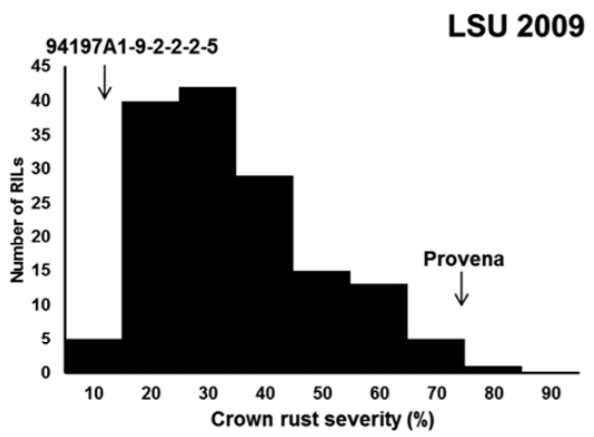

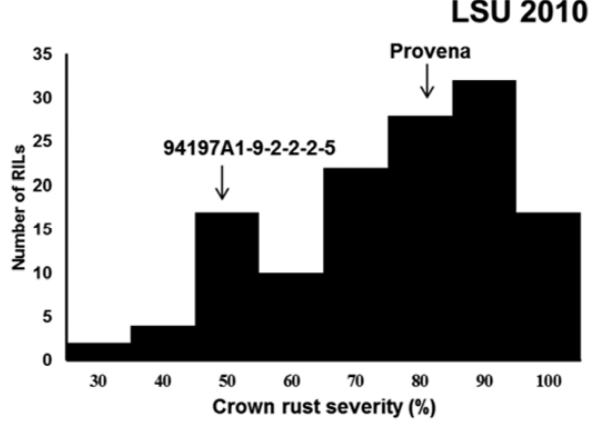

Fig. 2. Frequency distribution of oat crown rust severity in recombinant inbred lines (RILs) from a cross between Provena and 94197A1-9-2-2-2-5 tested in five field trials conducted at the Cereal Disease Laboratory (CDL) in St. Paul, MN, and at Louisiana State University (LSU) in Baton Rouge, LA. 
interval flanked by four SNP markers, and it explained 10 to $15 \%$ of the phenotypic variation. This QTL was nearly detected in the 2009 LSU test with the two markers with LOD 2.5 and 2.6, slightly below the threshold of significance. From the 2009 CDL trial, QCr.cdl9lsu9-19A was detected in a 15.8-cM interval flanked by six SNP markers, and it explained 11 to $20 \%$ of the phenotypic variation (Table 2; Fig. 3). This QTL was also detected in the 2009 LSU test with the three significance markers explaining 10 to $12 \%$ of the phenotypic variation (Table 2; Fig. 3). QCr.cdl9-12D was detected in a 15.4-cM interval spanned by four SNP markers that explained 10 to $11 \%$ of the phenotypic variation (Table 2; Fig. 3). Using means from all five environments, QCr.cdl9-19A, QCr.cdl9lsu9-19A, and QCr.cdl9-12D were identified. Using the five-trial mean, $Q C r . c d l 9$-19A explained 10 to $13 \%$ of the phenotypic variation and was spanned by four SNP markers with LOD scores ranging from 3.1 to 4 . QCr.cdl9lsu9$19 A$ explained 10 to $15 \%$ of the phenotypic variation and was spanned by five SNP markers with LOD scores ranging from 3 to 4.8. QCr.cdl9-12D explained 10 to $12 \%$ of the phenotypic variation and was spanned by four SNP markers with LOD scores ranging from 3.2 to 3.7. All QTLs were conferred by alleles from the partially resistant parent CDC Boyer (Table 2).

Provena/94197A1-9-2-2-2-5. In the P9 population, 1,053 polymorphic markers were used to construct the linkage map. Using the recombination and linkage groups and the linkage map order functions in JMP Genomics, 889 SNP markers were anchored to 41 linkage groups representing 21 oat chromosomes with a total map length of $1,022.5 \mathrm{cM}$. Using the $2011 \mathrm{CDL}$ test data, CIM analysis revealed the presence of one QTL for PR to crown rust in a 9.7-cM interval on chromosome 13A identified by five SNP and designated QCr.cdl11-13A. This QTL explained 19 to $35 \%$ of the phenotypic variation and was conferred by alleles from the resistant parent 94197A1-9-2-2-2-5 (Table 2; Fig. 4).

QTL validation. Eighty RILs from the 9B population were used to validate the QTLs that were detected in the two populations developed from the crosses with Provena. Of 5,744 SNP markers assayed, 1,119 polymorphic SNPs were used to construct a linkage map. Using recombination and linkage groups and the linkage map order functions, 934 SNP markers were assigned to 21 oat chromosomes with a total map length of $937.9 \mathrm{cM}$. One QTL was detected on chromosome 13A from the 2011 CDL screening test in an 8.1-cM interval. This QTL was identified by five SNP markers that explained 28 to $53 \%$ of the phenotypic variation with LOD scores ranging from 4.6 to 10.4 . This QTL was also detected in the 2009 CDL test with two significant markers explaining 18 to $19 \%$ of the phenotypic variation with LOD scores of 3.4 and 3.5. Using means from all five environments, QCr.cdl9-11-13A was detected with two significant SNP markers with LOD scores ranging from 3.3 to 3.9 and explaining 18 to $21 \%$ of the phenotypic variation. As in the P9 population, the alleles associated with resistance were contributed by 94197A1-92-2-2-5.

Sequence homology. Sequences of the significant SNP markers (13) were compared with the rice genome to identify potential candidate gene(s) for resistance within the QTL regions. The SNP markers, GMI_ES02_c3359_447, GMI_ES15_c3200_563, and GMI_ES15_c6639_318, linked to the QTL on 12D in PB, showed significant sequence homology with the rice sequences LOC_Os04g56620, LOC_Os04g42270, and LOC_Os04g39270 on chromosome 4 encoding for a molybdopterin biosynthesis protein, a $60 \mathrm{~S}$ ribosomal protein, and indole-3-glycerol phosphate synthase, respectively. These three sequences spanned a 17,350$\mathrm{Kb}$ region on chromosome 4 of rice, which is homologous to chromosome 12D of oat (13). Using the genome browser, eight candidate genes for resistance were identified within this region. Five of these are NBS-LRR genes (LOC_Os04g52970, LOC_Os04g53030, LOC_Os04g53060, LOC_Os04g53160, and LOC_Os04g53496), two are RPP genes (LOC_Os04g43340 and LOC_Os04g53050), and one is an RPM gene (LOC_Os04g46300). In the P9 population, four of the markers associated with QCr.cdl11-13A QTL on chromosome 13A showed significant sequence homology to locus on chromosome 6 of rice. This region

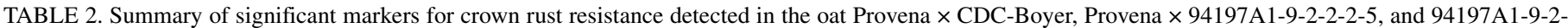

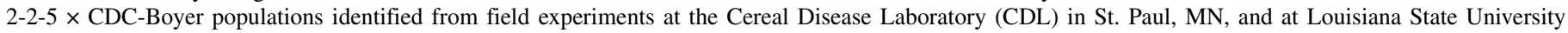
(LSU) in Baton Rouge, LA ${ }^{a}$

\begin{tabular}{|c|c|c|c|c|c|c|c|}
\hline Population & QTL & Marker & Chromosome & Environment & LOD & $R^{2}(\%)$ & Additive effect \\
\hline \multirow[t]{17}{*}{ Provena $\times$ CDC-Boyer } & \multirow[t]{9}{*}{ QCr.cdl9-19A } & GMI_DS_LB_10834 & $19 \mathrm{~A}$ & CDL-09 & 3.6 & 11 & -3.9 \\
\hline & & GMI_ES02_c27120_208 & $19 \mathrm{~A}$ & CDL-09 & 3.3 & 10 & -3.8 \\
\hline & & GMI_ES03_c6181_441 & $19 \mathrm{~A}$ & CDL-09 & 4.9 & 15 & -4.1 \\
\hline & & GMI_ES15_1rc9062_227 & $19 \mathrm{~A}$ & CDL-09 & 4.1 & 13 & -3.9 \\
\hline & & GMI_ES02_c6122_167 & $19 \mathrm{~A}$ & CDL-09 & 4.5 & 14 & -4.1 \\
\hline & & GMI_ES03_c10194_367 & $19 \mathrm{~A}$ & CDL-09 & 3.5 & 11 & -3.6 \\
\hline & & GMI_ES05_c5211_395 & $19 \mathrm{~A}$ & CDL-09 & 5.1 & 16 & -4.4 \\
\hline & & GMI_DS_cc11093_89 & $19 \mathrm{~A}$ & CDL-09 & 6.5 & 20 & -4.7 \\
\hline & & GMI_ES05_c83_464 & $19 \mathrm{~A}$ & CDL-09 & 5.9 & 18 & -4.5 \\
\hline & \multirow[t]{5}{*}{ QCr.cdl9lsu9-19A } & GMI_ES22_c721_179 & $19 \mathrm{~A}$ & CDL-09 & 3.7 & 11 & -3.7 \\
\hline & & GMI_ES02_c6122_167 & $19 \mathrm{~A}$ & LSU-09 & 3.7 & 12 & -4.2 \\
\hline & & GMI_DS_cc11093_89 & $19 \mathrm{~A}$ & LSU-09 & 3.0 & 10 & -3.7 \\
\hline & & GMI_ES05_c83_464 & $19 \mathrm{~A}$ & LSU-09 & 3.3 & 10 & -3.9 \\
\hline & & GMI_ES02_c3359_447 & $12 \mathrm{D}$ & CDL-09 & 3.2 & 10 & -3.4 \\
\hline & \multirow[t]{3}{*}{$Q C r . c d l 9-12 D$} & GMI_ES15_c3200_563 & $12 \mathrm{D}$ & CDL-09 & 3.1 & 10 & -3.2 \\
\hline & & GMI_GBS_821 & $12 \mathrm{D}$ & CDL-09 & 3.3 & 11 & -3.3 \\
\hline & & GMI_ES15_c6639_318 & $12 \mathrm{D}$ & CDL-09 & 3.0 & 10 & -3.3 \\
\hline \multirow[t]{5}{*}{ Provena $\times$ 94197A1-9-2-2-2-5 } & \multirow[t]{5}{*}{$Q C r . c d l 11-13 A$} & GMI_ES15_c1630_786 & $13 \mathrm{~A}$ & CDL-11 & 11.2 & 33 & -7.9 \\
\hline & & GMI_ES01_1rc22746_326 & $13 \mathrm{~A}$ & CDL-11 & 12.0 & 35 & -8.0 \\
\hline & & GMI_ES_CC13970_83 & $13 \mathrm{~A}$ & CDL-11 & 8.8 & 27 & -0.2 \\
\hline & & GMI_ES17_c1629_493 & $13 \mathrm{~A}$ & CDL-11 & 7.0 & 22 & -6.5 \\
\hline & & GMI_DS_A3_37_143 & $13 \mathrm{~A}$ & CDL-11 & 5.8 & 19 & -6.0 \\
\hline \multirow[t]{7}{*}{ CDC Boyer $\times$ 94197A1-9-2-2-2-5 } & \multirow[t]{5}{*}{$Q C r . c d l 11-13 A$} & GMI_ES15_c1630_786 & $13 \mathrm{~A}$ & CDL-11 & 10.4 & 53 & -12.9 \\
\hline & & GMI_ES17_c3808_324 & $13 \mathrm{~A}$ & CDL-11 & 9.7 & 50 & -12.5 \\
\hline & & GMI_ES05_c14988_223 & $13 \mathrm{~A}$ & CDL-11 & 5.3 & 32 & -10.1 \\
\hline & & GMI_ES22_c11318_631 & $13 \mathrm{~A}$ & CDL-11 & 4.7 & 29 & -9.8 \\
\hline & & GMI_ES01_c27692_191 & $13 \mathrm{~A}$ & CDL-11 & 4.6 & 28 & -9.9 \\
\hline & \multirow{2}{*}{ QCr.cdl9-13A } & GMI_ES15_c1630_786 & $13 \mathrm{~A}$ & CDL-09 & 3.5 & 19 & -6.1 \\
\hline & & GMI_ES17_c3808_324 & $13 \mathrm{~A}$ & CDL-09 & 3.4 & 18 & -6.0 \\
\hline
\end{tabular}

${ }^{\text {a }} \mathrm{QTL}=$ quantitative trait locus, $\mathrm{LOD}=$ logarithm of odds. 
of rice chromosome 6 shows extensive orthology with several SNP markers on chromosome 13A of oat (13). SNP marker sequences GMI_ES_CC13970_83 and GMI_ES15_c1630_786 were homologous with rice loci LOC_Os06g10560.1 and LOC_Os06g05090, which encode a leaf-senescence-related protein and a methyltransferase-domain-containing protein, respectively. These two loci spanned a 5,470-kb region on chromosome $6 \mathrm{G}$ of rice. Within this region, there are seven candidate genes for resistance. Six of these are NBS-LRR genes (LOC_Os06g05359, LOC_Os06g06380, LOC_Os06g06390, LOC_Os06g06400, LOC_Os06g06410 and LOC_Os06g06850), and one is an LRR receptor kinase gene (LOC_Os06g08690).

\section{DISCUSSION}

Development of molecular markers closely linked to QTLs for partial resistance to oat crown rust is a prerequisite for MAS. Such selection would benefit efforts to manage crown rust by simplifying resistance breeding, since it would enable selection at the seedling stage for a trait that can otherwise be reliably identified only through rating adult plants for resistance in multilocation field trials (7). In addition, PR could be pyramided with major genes for resistance only through marker-assisted breeding, since major genes mask the PR phenotype. QTLs for resistance to oat crown rust have been identified in several mapping popu- lations using primarily amplified fragment-length polymorphism and restriction fragment length polymorphism markers $(1,3,9$, $10,16,20,21)$. Such markers are not well-suited to marker-assisted breeding. The present study is the first to use newly developed oat SNP markers and the associated physically anchored map of hexaploid oat to map crown rust PR in adult plants. Using these resources, four QTLs for PR were mapped to specific oat chromosomes, with three QTLs (QCr.cdl9-12D, QCr.cdl9-19A, and QCr.cdl9lsu9-19A) originating from the oat cultivar CDC Boyer and one (QCr.cdl11-13A) from the breeding line 94197A1-9-2-2$2-5$.

The QTL QCr.cdl11-13A was identified not only in the cross with Provena, but also in the cross between the two resistant parents using a smaller subset of 80 RILs. In the cross between the two resistant parents (B9), the QTL QCr.cdl11-13A was identified based on two of the CDL field trials. This result provides validation that the resistant QTL allele from 94197A1-9$2-2-2-5$ is effective in two different genetic backgrounds. In contrast, none of the QTLs detected in the PB population was validated in the $\mathrm{B} 9$ population. This result could be attributed to the size of the B9 mapping population and to the small effect of the detected QTLs on chromosomes 12D and 19A. QTLs identified based on data from more than one environment would be good candidates for further work to identify flanking markers and validate in additional genetic backgrounds. In addition, mapping
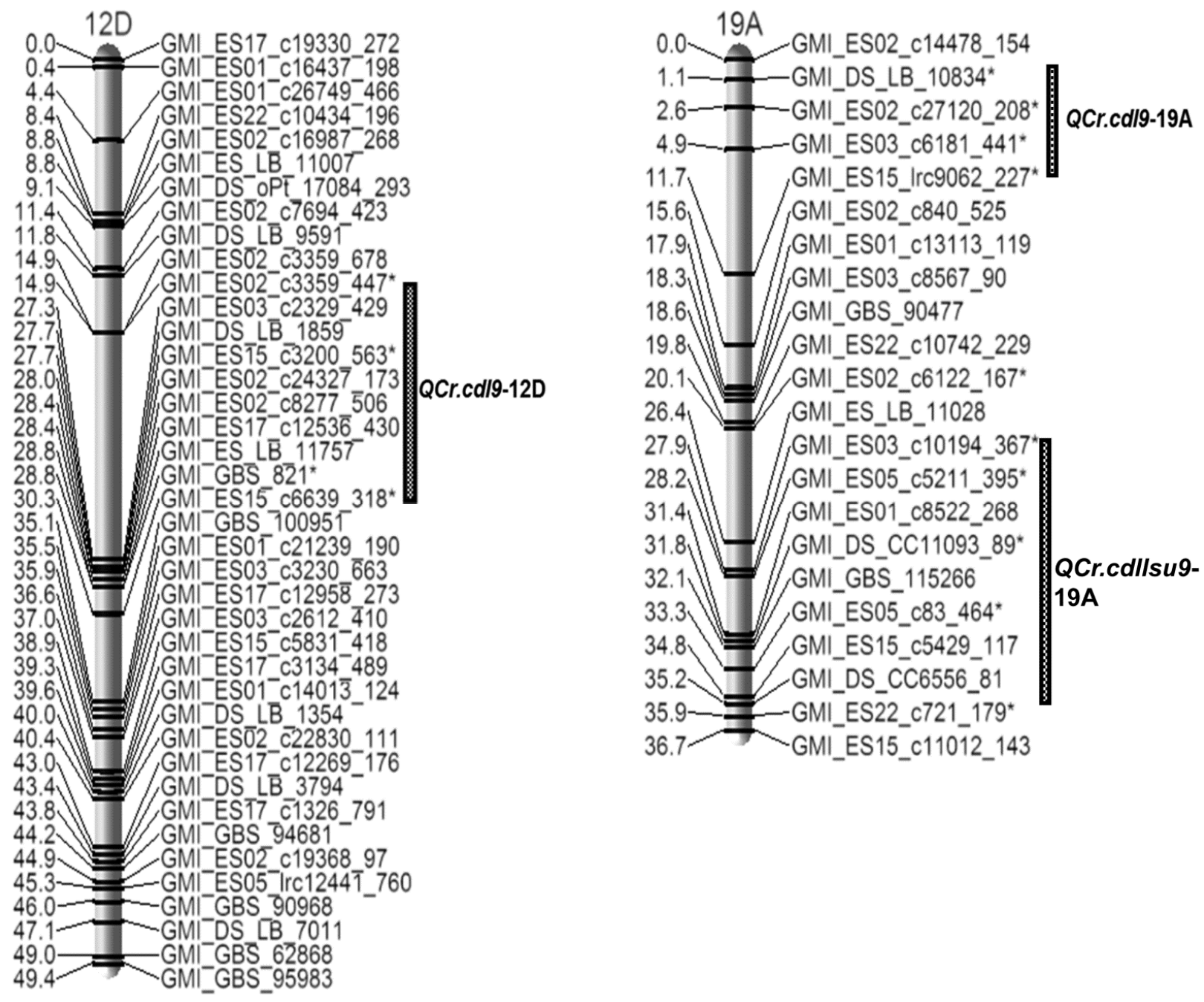

Fig. 3. Genetic linkage map constructed with single nucleotide polymorphism markers and 148 recombinant inbred lines derived from a cross between oat cultivars Provena and CDC-Boyer for chromosomes 12D and 19A showing quantitative trait loci for crown rust resistance identified from field experiments conducted at the Cereal Disease Laboratory (CDL) in St. Paul, MN, and at Louisiana State University (LSU) in Baton Rouge, LA. Asterisk (*) denotes a significant marker. 
populations that were previously studied, such as the Ogle/TAM O-301 and MN841801-1/Noble-2 populations, should be genotyped with the SNP panel in an attempt to find flanking SNPs for other important QTLs for crown rust PR. In 2010, disease severity at both the CDL and LSU was higher than in the other trials, and QTLs for PR were not detected in any of the populations using 2010 data. This result is probably due to the higher disease pressure reducing the difference in disease levels between PR and susceptible lines. The small plots used in the present study probably underestimated the effects of PR due to interplot interference.

The availability of the rice genome database as well as oat SNP sequences provides valuable tools for identification of candidate genes underlying the detected QTLs. To find candidate genes for resistance within each of the detected QTLs, the significant SNP marker sequences were used as queries for BLASTn searches against the rice genome database. Fifteen candidate genes were identified on chromosomes 4 and 6 of rice. Synteny between oat chromosome 12D and rice chromosome 4 as well as between chromosome 13A of oat and chromosome 6 of rice was reported (13). These candidate genes belong to NBS-LRR, and RPP, and RPM1 classes, which could be good targets for cloning from the parents used in the present study in an effort to find candidate resistance genes underlying this QTL. This finding could benefit the precision of MAS and may facilitate synteny-based positional cloning of disease resistance genes from oat.

Most of the markers in the present study mapped to the same chromosome as assigned in the consensus map generated by Oliver et al. (13). However, several markers that were previously mapped to chromosome $1 \mathrm{C}$ and $6 \mathrm{C}$ were grouped with markers from chromosome 7C-17A based on the $\mathrm{PB}$ and $\mathrm{P} 9$ populations. These differences could be due to the complex genome organization in oat and the presence of reciprocal translocations $(11,13$, 19). A relative lack of markers for chromosome $10 \mathrm{D}$ was observed in the PB population. This result could be attributed to the

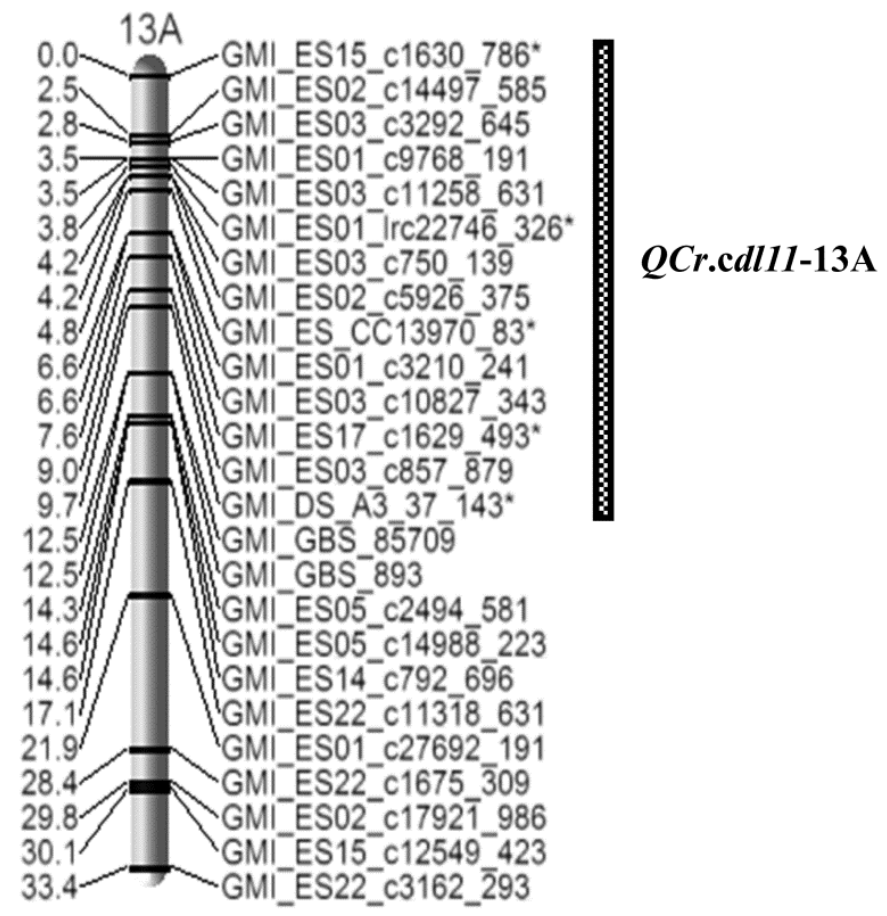

Fig. 4. Genetic linkage map constructed with single nucleotide polymorphism markers and 148 recombinant inbred lines derived from a cross between oat cultivar Provena and oat breeding line 94197A1-9-2-2-2-5 for chromosome 13A showing a quantitative trait locus for crown rust resistance identified from field experiment conducted at the Cereal Disease Laboratory (CDL) in St. Paul, MN. Asterisk (*) denotes a significant marker. lower rate of polymorphism in the D genome, which was observed also by Oliver et al. (13). As result, more QTLs on this chromosome may remain undetected.

In conclusion, using newly developed SNP markers and phenotypic data from field experiments conducted under conditions highly conducive to crown rust, we identified four QTLs for adult plant PR from CDC Boyer and 94197A1-9-2-2-2-5. The availability of SNPs with known map locations enabled for the first time identification of the chromosomal locations of QTLs for adult plant PR. The SNP markers linked to PR identified in the present study will provide a basis for further validation studies, implementation of marker-assisted breeding for PR to crown rust, and eventually identification of candidate genes underlying these QTLs.

\section{ACKNOWLEDGMENTS}

We thank N. Tinker for his valuable input and comments on this study and J. Avant, A. Sturbaum, and A. Frazier for technical assistance.

\section{LITERATURE CITED}

1. Acevedo, M., Jackson, E. W., Chong, J., Rines, H. W., Harrison, S., and Bonman, J. M. 2010. Identification and validation of quantitative trait loci for partial resistance to crown rust in oat. Phytopathology 100:511-521.

2. Anderson, J. A., Ogihara, Y., Sorrells, M. E., and Tanksley, S. D. 1992. Development of a chromosomal arm map for wheat based on RFLP markers. Theor. Appl. Genet. 83:1035-1043.

3. Barbosa, M. M., Federizzi, L. C., Milach, S. C. K., Martinelli, J. A., and Thome, G. C. 2006. Molecular mapping and identification of QTL's associated to oat crown rust partial resistance. Euphytica 150:257-269.

4. Bonman, J. M., Barnett, R. D., Chong, J., Harrison, S. A., Herrington, R., Jannink, J., McMullen, M. S., Obert, D. E., and Stuthman, D. D. 2004. Multi-location testing to identify oat germplasm with partial resistance to crown rust. Pages 177-178 in: 7th Oat International Conference Proceedings. P. Peltonen-Sainio and M. Topi-Hulmi, eds. MTT Agrifood Research Finland, Jokioinen, Finland.

5. Brake, V. M., and Irwin, J. A. G. 1992. Partial resistance of oats to $P$. coronata f. sp. avenae. Aust. J. Agric. Res. 43:1217-1227.

6. Chong, J., and Kolmer, J. A. 1993. Virulence dynamics and phenotypic diversity of Puccinia coronata f. sp. avenae in Canada from 1974 to 1990. Can. J. Bot. 71:248-255.

7. Collard, B. C. Y., and Mackill, D. J. 2008. Marker-assisted selection: An approach for precision plant breeding in the twenty-first century. Phil. Trans. R. Soc. B. 363:557-572.

8. Erickson, C. A., Wesenberg, D. M., Burrup, D. E., Whitmore, J. C., and Marshall, H. G. 2003. Registration of 'Provena' oat. Crop Sci. 43:23012302.

9. Jackson, E. J., Obert, D. E., Menz, M., Hu, G., and Bonman, J. M. 2008. Qualitative and quantitative trait loci conditioning resistance to Puccinia coronata pathotypes NQMG and LGCG in the oat (Avena sativa L.) cultivars Ogle and TAM O-301. Theor. Appl. Genet. 116:517-527.

10. Jackson, E. W., Obert, D. E., Avant, J. B., Harrison, S. A., Chong, J., Carson, M. L., and Bonman, J. M. 2010. Quantitative trait loci in the Ogle/TAM O-301 oat mapping population controlling resistance to Puccinia coronata in the field. Phytopathology 100:484-492.

11. Jellen, E. N., Gill, B. S., and Cox, T. S. 1994. Genomic in situ hybridization differentiates between A/D- and C-genome chromatin and detects intergenomic translocations in polyploid oat species (genus Avena). Genome 37:613-618.

12. Leonard, K. J. 2002. Oat lines with effective adult plant resistance to crown rust. Plant Dis. 86:593-598.

13. Oliver, R., Chao, S., Lazo, G., Tinker, N., Jellen, E. N., Carson, M. L., Rines, H. W., Bonman, J. M., Lutz, J. D., Shackelford, I., Korol, A. B., Obert, D. E., Wight, C. P., Gardner, K., Hattori, J., Beattie, A. D., Bjornstad, A., Jannink, J.-L., Brown-Guedira, G. L., Anderson, J. A., Fetch, Jr., Harrison, S. A., Howarth, C. J., Ibrahim, A., Kaeppler, H. F., Kolb, F. L., McMullen, M. S., Murphy, J. P., Ohm, H. W., Rossnagel, B. G., Yan, W. G., Miclaus, K. J., Hiller, J., Maughan, P. J., Redman, R. R., Islamovic, E., and Jackson. E. W. 2013. SNP discovery and chromosome anchoring provide the first physically-anchored hexaploid oat map and reveal synteny with model species. PLoS One 8(3):e58068.

14. Oliver, R. E., Lazo, G. R., Lutz, J. D., Rubenfield, M. J., Tinker, N. A., Anderson, J. M., Wisniewski-Morehead N. H., Adhikary, D., Jellen, E. N., Maughan, P. J., Brown-Guedira, G. L., Chao, S., Beattie, A. D., Carson, M. L., Rines, H. W., Obert, D. E., Bonman, J. M., and Jackson, E. W. 2011. 
Model SNP development for complex genomes based on hexaploid oat using high-throughput 454 sequencing technology. BMC Genomics 12:77.

15. Peterson, R. F., Campbell, A. B., and Hannah, A. E. 1948. A diagrammatic scale for estimating rust intensity on leaves and stems of cereals. Can. J. Res. 26:496-500.

16. Portyanko, V. A., Chen, G., Rines, H. W., Phillips, R. L., Leonard, K. J., Ochocki, G. E., and Stuthman, D. D. 2005. Quantitative trait loci for partial resistance to crown rust, Puccinia coronata, in cultivated oat, Avena sativa L. Theor. Appl. Genet. 111:313-324.

17. Rines, H. W., Molnar, S. J., Tinker, N. A., and Phillips, R. L. 2006. Oat. Pages 211-242 in: Genome Mapping and Molecular Breeding in Plants. Vol. 1: Cereals and Millets. C. Kole, ed. Springer, New York.

18. Simons, M. D., Martens, J. W., McKenzie, R. I. H., Nishiyama, I.,
Sadanaga, K., Sebesta, J., and Thomas, H. 1978. Oats: A standard system of nomenclature for genes and chromosomes and catalog of genes governing characters. U.S. Dep. Agric. Handb. 509.

19. Wooten, D. R., Livingston, D. P., Jellen, E. N., Boren, K. J., Marshall, D. S., and Murphy, J. P. 2007. An intergenomic reciprocal translocation associated with oat winter hardiness component traits. Crop Sci. 47:18321840 .

20. Wight, C. P., O’Donoughue, L. S., Chong, J., Tinker, N. A., and Molnar, S. J. 2004. Discovery, localization, and sequence characterization of molecular markers for the crown rust resistance genes $\mathrm{Pc} 38, \mathrm{Pc} 39$, and Pc48 in cultivated oat (Avena sativa L.). Mol. Breed. 14:349-361.

21. Zhu, S., and Kaeppler, H. F. 2003. Identification of quantitative trait loci for resistance to crown rust in oat line MAM17-5. Crop Sci. 43:358-366. 